\title{
Pre-juicios a la hora de pensar la nueva patología psiquiátrica
}

RESUMEN: Se señalan algunos usos y abusos extendidos del diagnóstico y de la clasificación y se propone prudencia y estudio a la hora de postular la existencia de nuevas estructuras clínicas, además de la neurosis y la psicosis.

PALABRAS CLAVE: Clasificación, diagnóstico, psiquiatría, psicoanálisis, psicosis.
SUMMARY: Some widespread uses and abuses of diagnosis and classification are singled out. Discretion and study are suggested when it comes to postulating the existence of new clinic structures in addition to neurosis and psychosis. KEY WORDS: Classification, diagnosis, psychiatry, psychoanalysis, psychosis.

\section{Quis furor iste novus?}

Se dice que una cosa es tener ideas ocurrentes y otra pensar ${ }^{2}$. Nosotros tenemos buenos ejemplos en el campo de las psicosis, donde abundan los «ensayos de rigor» que, con demasiada frecuencia, muestran mucha más lógica que la utilizada por sus custodios.

Estas son unas ideas ocurridas a partir de lecturas y reflexiones acerca de lo que significa nombrar, clasificar y diagnosticar en psiquiatría donde, como en tantas otras actividades humanas, se utilizan lugares comunes para argumentar a partir de ellos y, sin embargo, apenas se piensa sobre ellos (Bourdieu, 1999). La idea misma de diagnóstico-clasificación -se dan juntos- es, ante todo, un lugar común, es decir, presupone un espacio clasificatorio compartido por los objetos a clasificar y, también, es un lugar común del pensar, generalmente impensado, con el que se argumenta. Karl Kraus supo verlo con ironía a principios del siglo XX: «El diagnóstico es una enfermedad muy extendida» (Kraus, 1982).

La hipótesis de partida en realidad son preguntas: ¿son las clasificaciones una suerte de prejuicios, axiomas o postulados no explicitados que se aceptan como algo necesario e inevitable hoy, más que nunca, a-crítica y empíricamente? ¿Son de fiar?

\section{La comodidad clasificatoria ${ }^{3}$}

«El Sr. Silk procuraba que sus hijos no sólo aprendieran a hablar con propiedad, sino también a pensar lógicamente, a describir, enumerar, analizar y clasificar» (Roth, 2005).

\footnotetext{
«QQué nueva locura es ésta?» (Virgilio, Eneida, V, 670).

M. Azaña, refiriéndose a Ortega.

Borges (1989).
} 
En principio, la tarea de clasificar se presenta como un apremio del pensamiento ante la posibilidad de que el caos gane terreno frente al orden. Se conforma así, de antemano, una suerte de Todo, de un Uno continente donde puede alojarse lo múltiple. Es un ejemplo de la nostalgia de absoluto ${ }^{4}$, que diría Steiner; una especie de pensamiento uno, que acompaña al pensamiento occidental de Jonia a $J_{e n a^{5}}$; la tentación totalitaria del pensamiento, que reúne las cosas según la lógica de lo Mismo y lo Otro. ¿Y si «el orden y el desorden fuesen dos palabras que designan por igual el azar»? (Perec, 2001). Desde pequeños, inmersos en el caldo del lenguaje, empezamos a separar las cosas agrupándolas en virtud de las apariencias, experiencias, constancias, latencias, reminiscencias, insistencias, presencias, ausencias, recurrencias, ocurrencias, reticencias, interferencias y, sobre todo, diferencias, a través de las palabras y sus significancias. Es un fenómeno determinado por el lenguaje, que origina a su vez la funesta manía que nos asalta cuando pensamos, es decir, siempre ${ }^{6}$. Es una condición apriorística, determinada por el lenguaje, una suerte de imperativo clasificatorio. Como si de una cosmogonía se tratara, este imperativo pone límite al caos. Pero, eso no evita que en ella puedan anidar el germen del dislate y de la locura más disparatada, el ordenamiento más serio, y también el más débil, aunque esté consensuado por una mayoría ${ }^{7}$. Esta posibilidad del disparate, inherente a toda clasificación, viene siendo puesto en evidencia por la literatura, empeñada desde siempre, en hablarnos de lo ficticio de la realidad y del medio decir en el que vienen dichas las verdades (Gracián, 1994) de la ficción que más nos interesa ${ }^{8}$.

\section{¿Dislates clasificatorios?}

Kierkegaard, para huir de la rutina aburrida de las clasificaciones por falta de magín, prefería cualquiera sílabo que lo tuviera. Siguiendo a un cómico de la época, dividía a los humanos en 1) oficiales, 2) deshollinadores y 3) quijotescas maritornes (Kierkegaard, 1976).

Es muy citado el inicio de un relato de Borges que dice: «... que el doctor Franz Kuhn atribuye a cierta enciclopedia china que se titula Emporio celestial de conocimientos benévolos. En sus remotas páginas está escrito que los animales se dividen en: a) pertenecientes al Emperador, b) embalsamados, c) amaestrados, d) lechones, e)

\footnotetext{
Steiner.

Rosenweig.

Freud y sus Gedanken inconscientes.

Es un alivio que Aristóteles también considerara, entre los lugares de la cantidad, los de la minoría.

Literatura y psicoanálisis coinciden: eso que llamamos realidad es en realidad una ficción.
} 
sirenas, f) fabulosos, g) perros sueltos, h) incluidos en esta clasificación, i) que se agitan como locos, $\mathrm{j}$ ) innumerables, $\mathrm{k}$ ) dibujados con un pincel finísimo de pelo de camello, l) etcétera, m) que acaban de romper un jarrón, n) que de lejos parecen moscas» (Borges, 1989). Este texto, que dio lugar a que Michel Foucault escribiera Las palabras y las cosas, provoca una risa que sacude y trastorna porque subvierte los planos y superficies de las clasificaciones habituales. Es impensable... pero ordena.

Una última referencia a Georges Perec y su manera de preguntar al orden por su orden (que bien podía ser otro sin dejar de ser orden). Nos dice de las formas de ordenar los libros, o los objetos que se apilan y apiñan sobre su mesa. Llama a la tarea de "agrupar, clasificar y ordenar» actividades de repliegue o de aproximación oblicua. Por último, nos presenta un insólito texto administrativo de la realidad oficial francesa de su tiempo, que clasifica a los animales en: a) animales sobre los que se hicieron apuestas, b) animales cuya caza está prohibida desde el 1. ${ }^{\circ}$ de abril hasta el 15 de septiembre, c) ballenas encalladas, d) animales cuya entrada en el territorio nacional está sometida a cuarentena, e) animales en copropiedad, f) animales disecados, g) etcétera, h) animales que pueden contagiar la lepra, i) perros lazarillos, j) animales beneficiarios de herencias importantes, $\mathrm{k}$ ) animales que pueden ser transportados en una cabina, 1) perros perdidos sin collar, m) asnos, n) yeguas presumiblemente preñadas (Perec, 2001).

Después de esto se nos cuela la inquietud en el cuerpo, aunque sigamos pensando que esto sólo ocurre en la ficción o en algún recodo disparatado del mundo, pero no en el fundamento de nuestra profesión. «Elaborar una lista da una engañosa impresión de orden» (Bellow, 2005). El anancástico -que no cesa-, las hace en su intento de ordenar la pulsión. «No hay nada más vacilante que la instauración de un orden de las cosas» (Foucault, 1985). Buscando «el orden que se da en las cosas como su ley interior», nos tropezamos con un orden determinado por el lenguaje, pero que genera la ilusión de haber estado ahí desde siempre, esperando ser enunciado. Sólo entonces respiramos hondo y eso basta para que nuestras conciencias se aquieten en su vicisitud.

Puestos a la reflexión de las clasificaciones nos tropezamos con verdades acerca de ellas que no siempre estamos dispuestos a aceptar. Las clasificaciones no encuentran sus objetos, sino que los producen, puesto que son históricas, y las nociones que utilizamos son el resultado de una larga elaboración a través del tiempo.

\section{Clasificar y calificar en psiquiatría}

La clasificación como espacio continente es una premisa del diagnóstico. Este proceder, generalmente aceptado, se ha convertido en muchos casos en una 
actividad banal con el que empieza y termina todo el interés por la clínica. En los manuales suele tener su lugar en la parte general, después de la semiología. Ésta es su piedra angular, su elaboración es histórica y se realiza a través de la constancia de los síntomas y signos -la base de los diagnósticos sincrónicos-, y del curso -en los diacrónicos-. Pero, a pesar de recubrir con ella la totalidad del ámbito de los fenómenos clínicos, todas las clasificaciones semiológicas tropezaron siempre con un resto inclasificable.

La llamada psiquiatría neurocientífica, al pretender constituirse como ciencia natural de las enfermedades mentales, se ve obligada a adquirir sus usos y costumbres. Debe tener una sistemática, una taxonomía y una nomenclatura donde a cada especie le corresponda un lugar exclusivo y excluyente 9 .

En el ámbito de la ciencia natural, comenzó primero en Botánica, con Linneo y Buffon -su crítico nominalista y precursor del evolucionismo-, y el proceder sistemático de agrupar primero los datos naturales, mediante la especie y el género, y la elaboración teórica posterior, con la clase y el orden ${ }^{10}$. Después, en zoología, los post-darwinianos, añadiendo a la morfología la consideración genealógica o filogenética, cerraron la necesaria etapa descriptiva y clasificatoria. Lo que ofrecía la naturaleza era captado sólo por la mirada de lo que se daba a ver.

La psiquiatría de los siglos XIX y XX, mientras avanzaba, fue dando lugar a múltiples sistemas clasificatorios que privilegiaban determinados rasgos, no siempre coincidentes, al tiempo que la terminología propuesta iba poniendo de manifiesto una babel de escuelas que no terminaban de entenderse. Pero, pese a ello, y cada cual traduciendo y reinterpretando en su lengua lo que otros decían, se fue admitiendo con matices un cierto consenso, muy general, en el terreno de la nosografía psiquiátrica. Todas y cada una de las nosografías, aspiraban a cumplir con ese imperativo clasificatorio del Todo, propio de la ciencia desde Aristóteles («sólo hay ciencia de lo universal»).

No se quiso, supo o pudo ver que todas estas desavenencias en el consenso no había que entenderlas sólo como vanas polémicas de escuelas y países, sino que, en lo concerniente a los humanos y su patología psiquiátrica, se tropezaba muy pronto con lo imposible de clasificar, con algo que hacía obstáculo a lo universal, sin poder ver qué es ni en qué consiste.

Se puede decir que, situando los fenómenos del lado de la Mirada, se topaba con lo imposible de clasificar, de ordenar, de nombrar. Faltaba una nueva teoría

9 Pero sin olvidar, ni tampoco confundir, que el espacio de la enfermedad no es el cuerpo, sino el espacio clasificatorio (Foucault, 1978); (Braunstein, 1980). «Las enfermedades son modelos explicativos de la realidad y no elementos constitutivos de ésta... no existen verdaderamente más que en el mundo de las ideas» (Grmeck, 1994).

10 Classis et Ordo sapientia, Genus et Specie naturae (Linneo). 
del síntoma. Hasta entonces se pensaba que el síntoma, lo que no funcionaba y que aparecía en el campo fenoménico, era la alteración de una función. Pero no existía ninguna teoría de la ciencia natural que dijera qué funciones tenía la mente humana. Se recurrió a los saberes de la filosofía (pensamiento, voluntad y sensibilidad) y a las escuelas del sentido común y de las facultades para empezar a delimitar el campo de las funciones «psíquicas» (pensamiento, afectos, voluntad, percepción, memoria, inteligencia, motricidad, etc.). Los «síntomas» empezaron a ser vistos como desorden de las pasiones, de la percepción, del pensamiento, de la voluntad y los impulsos, de la conciencia, de la inteligencia... Así y todo, este proceder condujo a callejones sin salida ${ }^{11}$. La Mirada seguía teniendo un punto ciego. No podía ver al no disponer de una teoría del síntoma apta para lograrlo. Ésta vino de la mano de un nuevo paradigma con Freud, cuando puso en juego la escucha de los sujetos hablando de su sufrimiento. De ahí surgió el síntoma como un saber reprimido, que encierra un significado cifrado para el propio sujeto y una satisfacción inconfesable. En esto consiste lo que hace obstáculo, lo más particular, la subjetividad, aquello que no es universalizable y que sólo puede tenerse en cuenta en el caso por caso.

No obstante, pasando el tiempo, entre las muchas clasificaciones que se propusieron terminó por imponerse la noción de unidades clínico-evolutivas (Falret) y el diagnóstico a través del curso y de los estados finales (Kahlbaum y Kraepelin). A principios del siglo XX, los delirios crónicos de la escuela francesa y la noción de esquizofrenia en la alemana, junto a la PMD (Baillarger, Falret y Kraepelin) ocupaban todo el espacio nosográfico de las psicosis. Por supuesto, también estaban los rebeldes (Hoche, Kleist, Jaspers) que cuestionaban las entidades, y los que seguían con la idea de la psicosis única. Estos avatares son de sobra conocidos.

En el terreno nosológico y nosográfico estamos ahora ante un nuevo revival de la importancia para el diagnóstico del tiempo medible, que no es la temporalidad más lógica. Es muy curioso que esto suceda en las sociedades de capitalismo tardío, donde el consumo de bienes de goce precisa de estimaciones del tiempo de consumo. También surge una edición avant la lettre, arrogante y asertórica, del «fin de la historia» fukuyamiana aplicada a la nosografía, con la idea de que estamos en el techo de la historia, cuando todo ya está hecho y sólo resta arreglar los flecos. Los manuales estadísticos, empíricos y a-teóricos son, en realidad, prontuarios de signos (ya no hay síntomas), nomenclaturas que preparan el terreno para la intervención terapéutica, fundamentalmente farmacológica. No hay estructuras

11 Las alucinaciones, por ejemplo, ¿son alteraciones de la percepción o del juicio? ¿qué diferencia delirio y alucinación? (ver Colina, 2001). 
clínicas, no hay psicosis, tampoco neurosis. Sólo trastornos que remiten insensiblemente a la idea de que lo trastornado es el cerebro estudiado por la Neurociencia. Pero, «el cerebro no es un órgano digno» ${ }^{12}$. La psicosis es un orden del sujeto, no es una cuestión de déficit, es un ensayo de rigor. Lo sabemos.

Los criterios en boga son estadísticos, la constatación de los síntomas se realiza a través de protocolos y escalas universales, que consagran la evidencia de la Cifra, el fetichismo cifrado o «tontería numérica» (Colina, 2007). Los manuales vienen acompañados de unos glosarios que permiten la catalogación de los síntomas que se proponen para buscar. No queda nada de la conspicua semiología extraída directamente de la clínica de los casos particulares. Sólo queda la ceniza de los nombres como letanías que nada dicen del sujeto que los soporta.

Aunque, no toda la psiquiatría es igual. Cada vez prospera más la tendencia que cuestiona el «ordenamiento epidemiológico-financiero» de la clínica ${ }^{13}$. En los servicios públicos de Salud Mental, en los lugares alejados del brillo que trae aparejado el discurso universitario, se siguen usando las nociones de psicosis y neurosis y se manejan otros esquemas diagnósticos, mucho más respetuosos con la tradición psiquiátrica clásica. En ellos, el campo de las psicosis se distribuye, grosso modo, en dos grandes troncos: típicas o clásicas y atípicas o nuevas formas de presentación (Álvarez, 2006):

- Entre las psicosis clásicas:

1) esquizofrenia y cuadros de la misma estirpe (demencia precoz, locuras discordantes, automatismo mental, psicosis alucinatorias crónicas) ${ }^{14}$.

2) delirios paranoicos y crónicos (delirio crónico de evolución sistemática, locuras razonantes, delirios de imaginación o parafrenia confabulante, delirios pasionales, delirios sensitivos) ${ }^{15}$.

3) el círculo de las PMD (melancolías delirantes y ansiosas, cuadros maníacos, locura maníaco-depresiva) ${ }^{16}$.

- Las llamadas «nuevas» formas de presentación de las psicosis, nombradas de diversas formas: atípicas, blancas, comunes, estabilizadas, límites, ordinarias, fronterizas, no desencadenadas, latentes, sociales, etc. estudiadas con especial

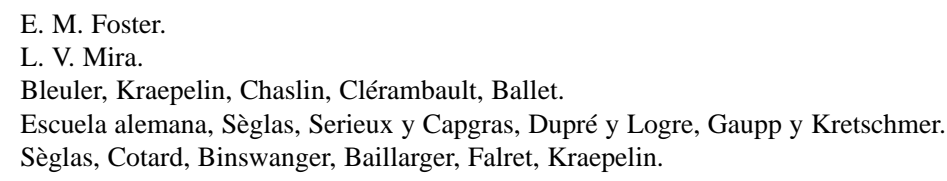


cuidado por el psicoanálisis y promocionadas, con ligereza y precocidad terapéutica, por cierta psiquiatría biológica. El debate nosográfico actual se polariza en este controvertido grupo.

\section{Consecuencias, (ab) usos y límites del diagnóstico}

Hemos visto que la clasificación crea los objetos de los que se ocupa. También crea un lenguaje, un modo de pensamiento y unas reglas semiológicas, pero, sobre todo, crea a «los psiquiatras como agentes de aplicación del sistema propuesto» (Braunstein, 1980). Al uniformizar el lenguaje y la formación, hace precisamente del psiquiatra «el soporte del discurso que lo ha constituido como tal». La pesadilla se muerde la cola y la pescadilla se repite.

Aún hay que considerar otros importantes aspectos del diagnóstico, el extraclínico (sociológico, económico y político), en la medida que lo que ordenan las modernas nomenclaturas son las demandas que un psiquiatra puede recibir, vengan de donde vengan ${ }^{17}$. Se le exige que realice una buena gestión de los recursos. Hay que recordar, otra vez, que el ordenamiento de la clínica actual -un ordenamiento que se ve demasiado-, evidencia su osamenta financiera y su olor a botica. En este sentido cumple su función social, pero no la clínica.

Existe una insoslayable dimensión ética en el diagnóstico que, como se ve, está presente en todos los ámbitos de la clínica. Esta dimensión afecta por igual tanto a quien lo realiza como a quien lo recibe.

Sabemos que la ética toma su referencia en el goce, y todo clínico debe saber del suyo como tal, el de la mirada, el narcisista de estar dentro de un grupo de elegidos, de hacerlo bien, etc. Quizá la teleología del diagnóstico sea la validación de la creencia en la existencia del Otro del Saber. En este sentido, el diagnóstico se presenta como rito, no sólo de iniciación o de paso, sino como liturgia, donde lo más importante es que quien toque a la puerta, por la razón que fuera, reciba como contrapartida un diagnóstico. Que se dicte un diagnóstico. Que se celebre el diagnóstico, que tal vez sólo exista para eso, para su celebración. Como si el diagnóstico existiese sólo para que pudiera realizarse y se realizara para garantizar su existencia. Vistas así las cosas, se puede decir, sin errar demasiado, que el diagnóstico psiquiátrico no tiene, ni quiere, tener nada que ver con la verdad, con la verdad implicada en la psicosis, en este caso. Se diagnostica al loco, al desviado, al potencialmente peligroso. Se califican ideas, sentimientos y conductas, etc. ${ }^{18}$ En

17 Ibíd.

18 Covarrubias, S. (2006), escribe a principios del siglo XVII: «Calificar proposiciones en el Tribunal del Santo Oficio, es declarar y demostrar que éstas son heréticas, erróneas o escandalosas». 
la práctica, se comporta como una sentencia. Se ve en todo ello, la finalidad de la adaptación social, que las cosas marchen, que el orden social no resulte amenazado. Calificar puede tener un uso protervo.

\section{Clasificar en psicoanálisis}

Los psicoanalistas siempre mostraron respeto por la clínica clásica. Entre ellos, sobresalen Freud y Lacan que nos enseñaron, partiendo de ella, a pensarla de otra manera. Podemos leer en ellos unas preciosas indicaciones acerca de los prejuicios. Freud, diciéndonos que no hay que apresurarse a encasillar, que hay que tomarse un tiempo para diagnosticar y partir cada vez desde cero. Comentándolo, Lacan ${ }^{19}$ aconsejaba tener especial cuidado en esto, porque lo propio de la experiencia es encasillar, recordando que lo único que tiene que saber el psicoanalista es olvidar lo que sabe, porque el saber de un sujeto con una estructura no es seguro que valga para otro del mismo tipo.

Merece, pues, destacarse que el psicoanálisis sólo concibe el uno por uno, el caso por caso, a pesar de que puedan formar serie.

El capítulo nosológico y nosográfico es escueto, parco. Fundamentalmente, neurosis y psicosis. Un eje fundamental de su teoría lo constituye el síntoma como efecto de lenguaje. En el caso de la neurosis, estructurado como un lenguaje, con una vertiente significante y otra de goce, con la represión y el retorno simbólico de lo reprimido; y la deslocalización de un exceso de excitación corporal que no pasa por el lenguaje, la angustia, lo simbólico realizado y la fragmentación de lo imaginario que no puede unificar tamaño desorden corporal, en el caso de la psicosis, por los efectos devastadores que la forclusión del Nombre del Padre tiene.

El criterio que orienta el diagnóstico psicoanalítico es un criterio estructural, que parte del fenómeno para llegar a la estructura. Los fenómenos son los aspectos fenomenológicos descritos por la psiquiatría clásica («la envoltura formal del síntoma»). La estructura, al contrario, no es un dato de lo observable, es algo que se deduce, se construye, algo que organiza lo observado. De tal manera que cualquier síntoma, en sentido general, es sólo una expresión fenomenológica de la estructura del sujeto determinado por el lenguaje. En este sentido, los fenómenos de la psicosis son índices de la estructura. Por eso debe precisarse qué tipo de estructura está en juego, evitando el riesgo de confundir el fenómeno con la estructura o hacer del fenómeno una nueva estructura (como ocu- 
rre con los borderline). La estructura es aquello de lo real que entra en el lenguaje y tiene, a su vez, algo más que la hace irreductible: la radical particularidad del sujeto, la posición subjetiva, el modo peculiar que cada uno tiene para hacer y vivir su locura (Puig, 1996). La estructura es un universal (Soler, 1994) surgido del discurso de la ciencia, ante la ineficacia de ésta para dar cuenta de los objetos de las denominadas «ciencias humanas» (sociología, lingüística, etnografía, etc.). Pero, es una estructura que admite lo peculiar, en tanto es incompleta, en tanto es «no-toda».

En psicoanálisis, el diagnóstico sólo tiene sentido dentro de una ética. No está interesado en normalizar ni las conductas ni los sujetos. Su interés está centrado sobre lo que dice un sujeto acerca de aquello que le pasa y en la brecha abierta en el inconsciente que esas mismas palabras posibilitan.

La única justificación del diagnóstico poco tiene que ver con los fines del encasillamiento en un universo clasificatorio. Cabe decir que cada caso ni es validado por el diagnóstico ni tampoco le da legitimidad a éste. Sólo encuentra su justificación en la clínica. «El saber clínico orienta la acción», saber cuáles son sus fenómenos, la coyuntura desencadenante, el trabajo del psicótico. Si no se sabe cómo está construida una psicosis, cuáles son sus condiciones y la naturaleza de sus fenómenos... se remará sobre la arena (Soler, 2005).

En el diagnóstico hay un juicio ético. Y este juicio merece ser considerado en varios aspectos, no sólo en lo que respecta a quien lo hace, sino por las consecuencias en quien lo sufre. Sin detenernos ahora en la banalización que cualquier etiqueta supone, es cierto que todo en la naturaleza tiene nombre y está clasificado. Sí, de acuerdo. Pero un león no sabe que él es un león. Lacan decía «todo significante injuria al sujeto», no por el significado que pueda tener, sino por la predicación misma por parte de otro. El significante aliena. El diagnóstico no debe funcionar nunca como si fuese un nombre propio. Hay siempre una violencia en el diagnóstico. No obstante, podemos discernir el tipo de violencia: la estrictamente necesaria e inevitable (la de toda predicación con un motivo clínico), que puede evitar estragos, o la gratuita y entreverada por intereses que están mucho más allá de los del sujeto diagnosticado.

Llegados a este punto, echando la vista atrás, las modernas nomenclaturas recuerdan al adagio de Chuang-Tzu, referido a las tareas imposibles: saltar más allá de la propia sombra o acallar el eco mediante un grito.

Así pues, las clasificaciones universales «deben leerse con astucia y cautela, es decir, a la defensiva $»^{20}$. En psiquiatría, acaso haríamos bien aplicando la

20 Extrapolación al asunto clasificatorio de la actitud que Bellow (2005) recomienda ante los medios de comunicación. 
navaja de Occam ${ }^{21}$, pero teniendo en cuenta la recomendación de Jaspers, afecto de un nihilismo diagnóstico sui generis, de que en el futuro de una psiquiatría especial, las unidades nosológicas llevasen por rótulos los nombres de cada paciente. Todos utilizamos esta manera de nombrar lo que se resiste a la clasificación.

\section{La psicosis en la clínica actual}

Se resumiría diciendo: Los que no tienen casillas sacan de las suyas a los psiquiatras. Hay prisa por clasificar porque el Amo nos impone su crono. Los clásicos, sin embargo, se tomaban su tiempo. Antes citaba el cambio en las clasificaciones por el cambio del Otro social. Pero, también hay cambios en la clínica por cambios en el Otro social, porque ha cambiado el discurso y los efectos de éste en los sujetos. Todo el mundo habla del cambio ocurrido socialmente en el mundo después de la caída del muro de Berlín, o después del 11-S. Pero, desde hace mucho, son legión quienes venían avisando del fuego que se avecinaba. Cada cual a su manera, desde Kafka, Freud, Benjamin, la escuela de Frankfurt, el existencialismo, los pensadores del holocausto, por nombrar a unos pocos.

Lacan habla del discurso capitalista que subvierte los discursos históricamente anteriores -actuantes aún hoy-, determinando un cambio de paradigma en el manejo de los goces (con la repercusión en la estructura libidinal de los sujetos, negando la castración y proponiendo un goce que la tapone, sin tener que pasar por el Otro, al tiempo que desfallece la función metafórica del padre). Sólo quiero apuntar esta referencia, porque tiene pertinencia en los cambios de la clínica de la psicosis. En otros ámbitos, son muchos los cambios que se ven ahora, junto a la actualidad renovada de viejos síntomas más generalizados (anorexia-bulimia, abuso de sustancias, patologías del acto). Lacan hablaba de «psicosis social», de sujetos con enfermedad mental «perfectamente normales», para referirse a esos sujetos psicóticos sin desencadenar una psicosis clásica, capaces de llevar una vida de lo más normal, prendidos a los emblemas del Otro, pero con «vacío existencial, experiencia de ausencia e insustancialidad anómica» (Recalcati, 2003). Signos, índices, síntomas, que plantean preguntas. Creo que la influencia del psicoanálisis en este campo es fundamental y será mayor en la medida que se estudie más la nueva clínica, permitiendo el desarrollo de los modelos psicoanalíticos (clínica borromea, la dialéctica de falta y vacío, la metáfora débil, las descompensaciones imaginarias).

21 Pluralitas non ponenda sine necesitate («No debe introducirse innecesariamente una pluralidad»). 
Antes hablaba de la precaución de no confundir forma con fondo, fenómeno con estructura, psicosis estabilizadas y nuevas psicosis, que generan falsas ilusiones que, en algunos casos, dan lugar a verdaderas habladurías nosográficas. Tampoco habrá que olvidar que lo imposible de clasificar volverá a colocarse en primer plano. La reflexión aplicada a la senda clínica es el único recurso. Acaso no se trate tanto de saber qué es, en el sentido de describir, nombrar y agrupar, sino de entender qué le pasa a alguien, cuáles son las condiciones de aparición, cómo responde a lo que le pasa. Las preguntas planteadas son, básicamente, dos: ¿son nuevas estructuras clínicas? (como propone O. Kernberg con los borderline) ¿son nuevas formas de presentación de las psicosis? Este terreno es apasionante y excede el ámbito de este trabajo. Mientras tanto, convendría recordar a Occam, a los clásicos y a Freud.

\section{BIBLIOGRAFÍA}

(1) Álvarez, J. M., «Psicosis actuales», Estudios sobre la psicosis, Vigo, AGSM, La Otra Psiquiatría, 2006.

(2) BACAllado, J. J., «Sobre nominación y clasificación», Discurso de Ingreso a la Academia Canaria de la Lengua, La Laguna, 2002.

(3) Bellow, S., Todo cuenta, Madrid, Galaxia Gutenberg-Círculo de Lectores, 2005.

(4) Borges, J. L., Otras inquisiciones, «El idioma analítico de John Wilkins», OC-I. Barcelona, RBA-Instituto Cervantes, 2005.

(5) Bourdieu, P., Contrafuegos, Barcelona, Anagrama, 1999.

(6) Braunstein, N., Psiquiatría, teoría del sujeto, psicoanálisis, México, Siglo XXI, 1980.

(7) Colina, F., El saber delirante, Madrid, Síntesis, 2001; y De locos, dioses, deseos y costumbres, Valladolid, El Pasaje de las Letras, 2007.

(8) Covarrubias, S., Tesoro de la lengua castellana o española, Madrid, CECE-RAE, 2006.

(9) Foucault, M., El nacimiento de la clínica, México, Siglo XXI, 1978; y Las palabras y las cosas, Barcelona, Planeta-De Agostini, 1985.

(10) Gmreck, M., Les maladies à l'aube de la civilisation occidental, París, Payot, 1994.

(11) Gracián, B., Arte de la prudencia. Oráculo manual, Madrid, Temas de Hoy, 1994.

(12) JASPERS, K., Psicopatología General, Buenos Aires, Beta, 1970.

(13) KierKegaARD, S., La repetición, Madrid, Guadarrama, 1976.

(14) Kraus, K., Contra los periodistas y otros contras, Madrid, Taurus, 1982.

(15) LACAN, L., «L'Etourdit», Escansión, 1, 1984, Barcelona.

(16) LaCAN, L., «Conferencia sobre el síntoma en Ginebra», Intervenciones y textos, 2, Buenos Aires, Manantial, 1988.

(17) Manchado, S., «De síntomas y diagnósticos», Alisios, 3-4, 1997, Las Palmas de Gran Canaria, Seminario Freudiano de las Islas Canarias.

(18) Perec, G., Pensar, clasificar, Barcelona, Gedisa, 2001.

(19) PUIG, M., y otros, «Aproximaciones al problema del diagnóstico», Notas Freudianas, 2, Gijón, GEAS, 1996. 
(20) Recalcati, M., Clínica del vacio, Madrid, Síntesis, 2003; y La última cena, Buenos Aires, Ediciones del Cifrado, 2004.

(21) Rотн, Ph., La mancha humana, Madrid, Alfaguara, 2005.

(22) Rosenweig, citado por Mate, R., Memoria de Auschwitz, Madrid, Trotta, 2003.

(23) Soler, C., «Los diagnósticos», Freudiana, 16, Escuela europea de psicoanálisis, Cataluña, 1996. «La querella de los diagnósticos», Formaciones clínicas del Campo Lacaniano, Colegio Clínico de París, Curso 2003-2004, 2005.

* Segundo Manchado Romero. Psiquiatra. Psicoanalista. Jefe de Servicio de Psiquiatría (Unidades Clínicas y Rehabilitación; Unidad de Media Estancia). Hospital Universitario de Gran Canaria Dr. Negrín.

Correspondencia: Segundo Manchado. Hospital Juan Carlos I, C/ Real del Castillo n. ${ }^{\circ}$ 152, Las Palmas de Gran Canaria, 35014. smanrom@ gobiernodecanarias.org

** Fecha de recepción: 8-III-2008 (aceptado el 14-IV-2008). 